\title{
Fundamental measurements with muons - View from PSI
}

\author{
Bernhard Lauss \\ Department of Particles and Matter - UCN Group, \\ Paul Scherrer Institut, CH-5232 Villigen-PSI, Switzerland
}

\begin{abstract}
Muons can serve as probes to precisely determine fundamental parameters of the Standard Model or search for 'new physics'. The high intensity muon beams at the Paul Scherrer Institut (PSI) allow for precision measurements and searches for rare or forbidden processes. Both types of experiments challenge the Standard Model in a way complementary to high energy physics. We give a short overview of recent results and ongoing experiments at PSI, and of ideas for the future.
\end{abstract}

Key words: muon physics, rare decay, muon lifetime, Fermi constant, muon capture PACS: $14.60 \mathrm{ef}, 13.35 \mathrm{Bv}, 12.15 .-\mathrm{y}, 21.45 . \mathrm{Bc}, 25.30 . \mathrm{Mr}, 13.15 .+\mathrm{g}$

\section{Introduction}

In 2008 the Paul Scherrer Institut (PSI) 1 celebrated its $20^{\text {th }}$ anniversary and many years of delivering high intensity muon beams. Several upgrades made the $590 \mathrm{MeV} / 51$ $\mathrm{MHz}$ ring cyclotron to be up to today the most powerful proton accelerator of its kind in the world, which delivers several $10^{8}$ muons per second to experiments. The accelerator runs now routinely with $2.0 \mathrm{~mA}$ proton current and was already pushed to $2.15 \mathrm{~mA}$ for tests. In the near future running at $2.3 \mathrm{~mA}$ is foreseen, and an extensive program was launched to boost the operating proton current to $2.6 \mathrm{~mA}$, by 2011 , and ultimately to 3.0 $\mathrm{mA}$, envisaged for 2012 [1]. Precision experiments should benefit from a correspondingly increased muon intensity.

\section{Search for the decay $\mu^{+} \rightarrow e^{+}+\gamma$}

Charged lepton-flavor conservation has been empirically verified to a high precision, but is not a consequence of a known underlying symmetry. The decay $\mu \rightarrow e \gamma$ is leptonflavor violating and hence, excluding neutrino flavor mixing, forbidden within the Standard Model (SM). Neutrino masses and mixing, which is established now, introduce a contribution to this decay within the SM, however, on an unmeasurably small level of order $\sim 10^{-55}[2]$. On the other hand, there are several attractive theories beyond the SM, such as supersymmetry, which generally predict lepton-flavor-violating processes at a level within today's experimental reach. A corresponding experimental signal would be free of SM background and hence a clear indication for 'new physics'.

\footnotetext{
Email address: bernhard.lauss@psi.ch (Bernhard Lauss).

1 www.psi.ch
}

Preprint submitted to Elsevier

3 May 2021 


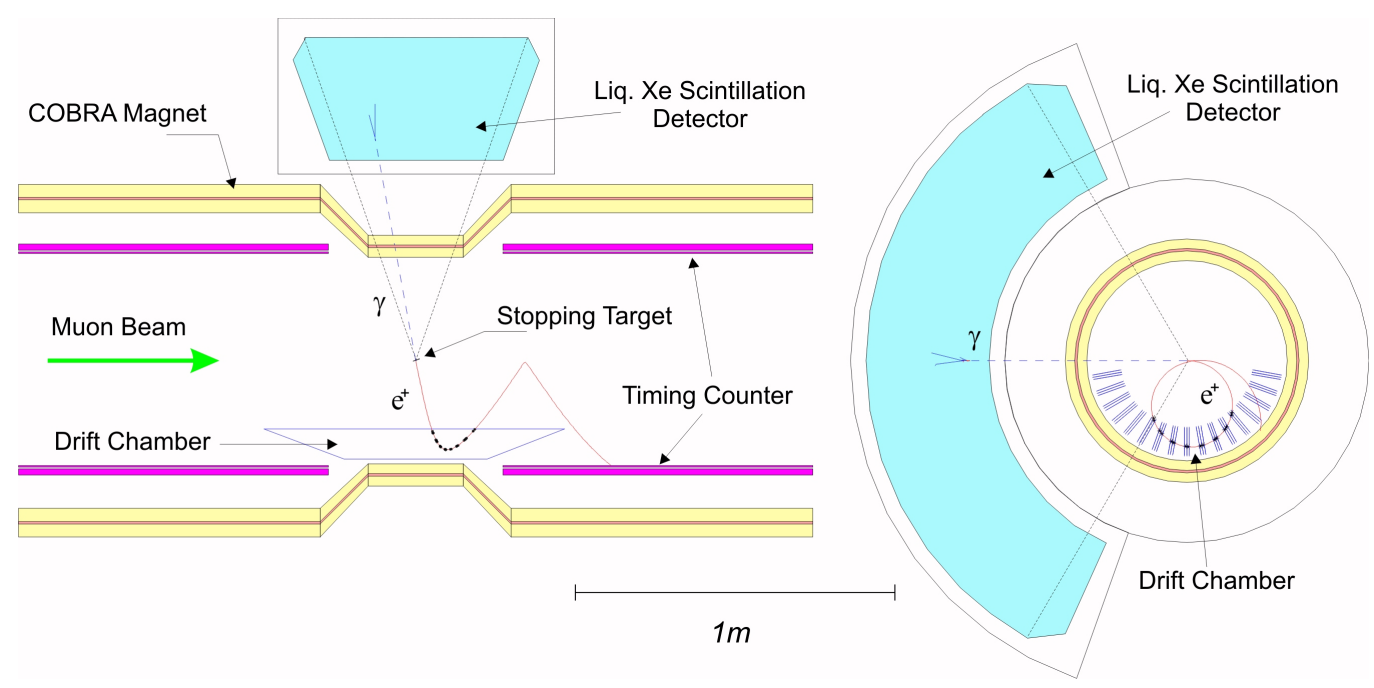

Fig. 1. Sketch of the MEG experiment's main components [2].

The goal of the MEG experiment at PSI [2] is to reach a sensitivity of $10^{-13}$, improving the present limit $[3$ by almost 2 orders of magnitude. Consequently one needs a detector managing a challenging high muon stop rate up to $10^{8}$ muons/s. The experimental principle is based on the simultaneous detection of the back-to-back emitted mono-energetic decay positron and gamma. The positrons are detected in high rate drift-chambers located in a magnetic field for momentum determination and in scintillation counters for timing. The gammas are detected in the world's largest liquid xenon scintillation counter, as sketched in Fig.1. Excellent timing, energy and spatial resolution for both reaction products are required to beat the main background caused by ordinary muon decay and pile-up. 2008 saw the first months of physics run of MEG and the accumulated statistics looks promising to already improve the present limit on $\mu \rightarrow e \gamma$ significantly.

\section{Muon lifetime measurements}

The Fermi constant $G_{F}$ describes the strength of the charged-current weak interaction. Along with the fine structure constant $\alpha$ and the $Z$-boson mass, it is one of the three pillars of the electroweak Standard Model and directly related to the electroweak gauge coupling [4. The most precise determination of $G_{F}$ is based on the mean lifetime of the positive muon, $\tau_{\mu}$, and can be extracted from:

$$
\frac{1}{\tau_{\mu}}=\frac{G_{F}^{2} m_{\mu}^{5}}{192 \pi^{3}}(1+\Delta q)
$$

with $\Delta q$ representing higher order QED and hadronic corrections as well as finite-leptonmass phase space factors, which have only recently been computed to a sub-ppm level 5]. A first computation of order $\alpha^{2}$ using a finite electron mass shifted the value of $\Delta q$ by another $0.43 \mathrm{ppm}$ 6. Hence, a comparably precise experimental determination of $\tau_{\mu}$ is highly desirable.

The MuLan experiment [7 installed a muon beam kicker on the PiE3 beamline at PSI, which allows after directing positive muons onto a target for a selectable time 

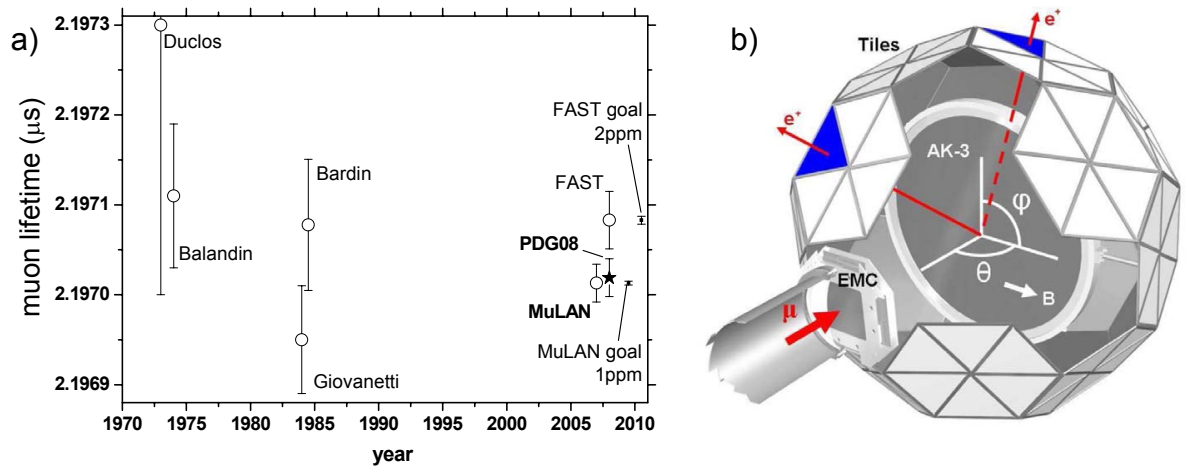

Fig. 2. a) The improvement of the muon lifetime over the last 40 years [7|8|9], together with the 2008 $P D G$ average (star) and the goals of the PSI experiments, with errors hardly visible on this scale. A 1 ppm error on $\tau_{\mu}$ translates into a $0.5 p p m$ error on $G_{F}$.

b) Sketch of the MuLan detector (EMC - entrance muon counter, AK-3 - the target disc, tiles - the parts of the scintillation counter) [7].

period (e.g. $5 \mu \mathrm{s}$ ), to steer away the beam for the following, for instance, $22 \mu \mathrm{s}$, The decay positrons are recorded in a soccer-ball shaped detector (see Fig2 2 ) made from 170 double-layer scintillator tiles, which are read out via custom-made $500 \mathrm{MHz}$ FADC modules able to separate pulse pile-up events on the ns level. Systematic issues, caused by positron detection differences in the counters, due to polarized muons precessing in the earth's magnetic field, are dealt with via measurements in different targets, which are in a homogeneous magnetic field and either fully maintain the muon polarization (silver), depolarize the muons to a large extent (sulphur), or cause a very fast muon precession due to an internal few Tesla high magnetic field (Arnokrome ${ }^{T M}-\mathrm{II}{ }^{2}$ ). Several $10^{12}$ muon decays were recorded for each target. The first MuLan result, based on part of the data has set a new precision benchmark, as shown in Fig $2 \mathrm{a}$. Additionally, several dedicated systematic measurements are presently under analysis. The final precision goal on $\tau_{\mu}$ is $1 \mathrm{ppm}$, which translates into a $0.5 \mathrm{ppm}$ precision on $G_{F}$.

The FAST experiment [8] relies on the detection of the full decay sequence $\pi \rightarrow \mu \rightarrow e$ and corresponding times in a fast imaging target made of $32 \times 48$ pixels, constructed from plastic scintillator bars in a homogeneous B field. This approach allows a good control of muon polarization effects. FAST is scheduled to achieve a statistics of several $10^{11}$ in 2008/2009. Its goal is a $2 \mathrm{ppm}$ measurement of $\tau_{\mu}$. As a by-product, FAST can also measure the $\pi^{+}$lifetime and improve the present world average.

\section{Muon capture measurements}

The determination of the proton's weak pseudoscalar coupling constant $g_{P}$ has been the driving force behind decades of muon capture measurements. The PSI result on the muon capture rate in ${ }^{3} \mathrm{He}[10$ has set a precision landmark in this field. However, with 3 involved nucleons some questions still remained in the precise theoretical prediction. A specially exciting turn came with the precise TRIUMF results from a measurement of radiative muon capture (RMC) in hydrogen [11, which disagreed with theory and

\footnotetext{
$\overline{2}$ a proprietary Fe-Cr-Co alloy of Arnold Engineering Co., Marengo, IL, USA
} 
a)

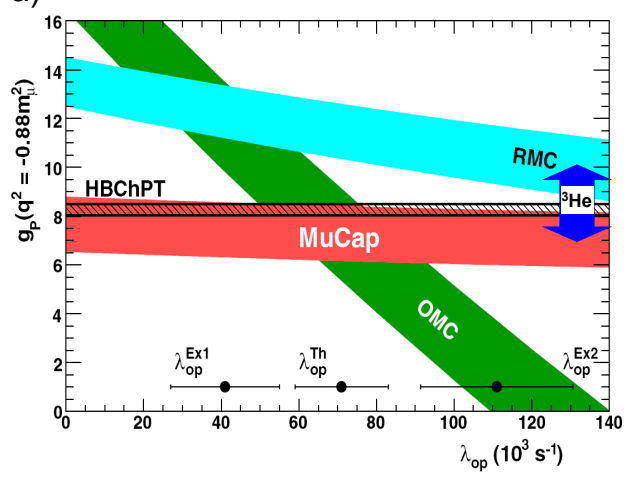

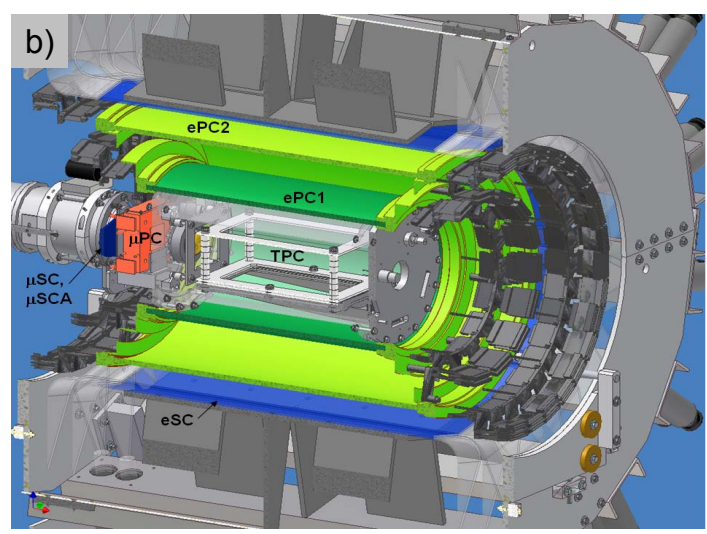

Fig. 3. a) Present knowledge on the proton's pseudoscalar coupling constant. Explanantion see text. b) The MuCap detector: $\mu S C / \mu P C$ - muon entrance counters, TPC - time projection chamber $=$ target, ePC, eSC - electron tracking and timing counters.

results derived from ordinary muon capture (OMC) measurements [14, as shown in Fig 3 . After decades of worldwide experimental efforts, the MuCap experiment has achieved the first unambiguous determination of the proton's pseudoscalar coupling $g_{P}$ 12 and has solved a longstanding discrepancy. The result is in excellent agreement with recent calculations based on heavy baryon chiral perturbation theory (HBChPT) [13. Experimental determinations of $g_{P}$ depend on the ortho-para transition rate $\lambda_{o p}$ in the p $\mu$ molecule. The most precise previous measurement of ordinary muon capture (OMC) [14] and the RMC experiment [1] both depend significantly on the value of $\lambda_{o p}$, which itself is poorly known due to mutually inconsistent experimental [15] and theoretical [16] results. In contrast, the first MuCap result for $g_{P}[12$ is almost independent of molecular effects.

The MuCap result was only possible with an enduring joint effort and a rigorous experimental technique [17. The setup is shown in Fig]3p. The active target, a time projection chamber, was filled with 10 bar of ultra-pure (high $Z$ contamination in the few ppb range) and isotopically pure hydrogen [18. Muon stops and corresponding decay electron tracks were recorded in 3 dimensions, which allowed for very selective cuts and hence an unprecedented control and possible study of systematic effects. Specifically, muon capture events on high $Z$ elements are even on the ppb contamination level visible in the MuCap detector. Target conditions were selected in order to control effects due to muonic molecule formation and muon catalyzed fusion. The muon capture rate was finally determined by comparing the lifetime of negative muons in hydrogen with the positive muon lifetime from the more precise result [7. In order to extract $g_{P}$, the singlet muon capture rate was compared to two recent calculations 19 adding the newly calculated radiative correction 20]. Presently, the full data set of more than $10^{10}$ recorded $\mu^{-}$stops in hydrogen is being analyzed in a blind analysis, with the final precision goal of $1 \%$ on the singlet muon capture rate in hydrogen.

Knowing $g_{P}$ from MuCap facilitates the interpretation of the doublet muon capture rate on the deuteron $\left(\Lambda_{d}\right)$, the measurement goal of the recently started MuSun experiment [17/22 23] which also aims at $1 \%$ precision. Such a result would allow a precise test of modern effective field theories and would represent the most stringent test of electro-weak interaction in a two-body system [21. Moreover, it would allow determi- 
a)

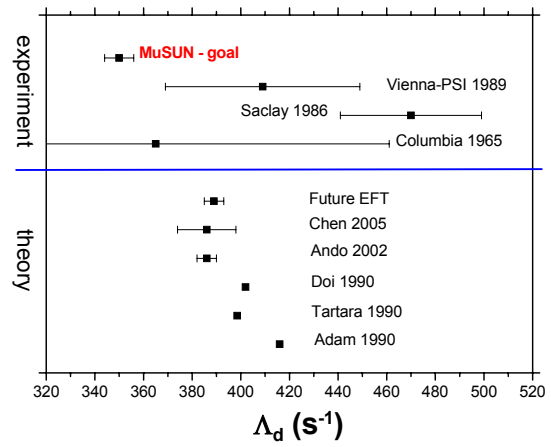

b)

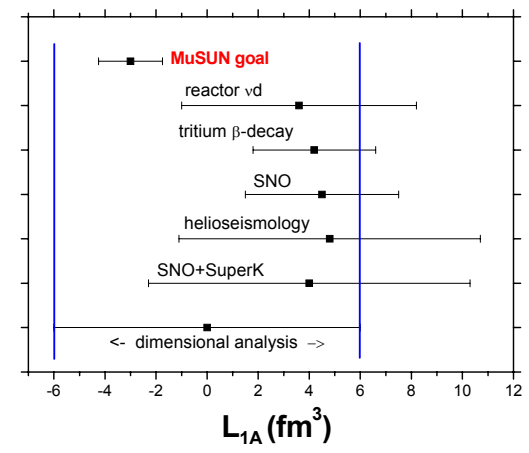

Fig. 4. a) Experimental determinations of the doublet muon capture rate on the deuteron in comparison with recent calculations. Meson exchange currents contribute $\sim 20 \mathrm{~s}^{-1}$ to the total rate. b) Estimations on the axial two-body current term $L_{1 A}$. All methods up to now include certain assumptions and approximations which might be questioned. All references are given in [22]

nation of the axial two-body current term which scales with the low energy constant denoted $L_{1 A}$ (or $\hat{\mathrm{d}}^{R}$ ) 21]. This parameter is of astrophysical interest, as it appears in the same form in the cross-section for i) muon capture on the deuteron, ii) $p p$ fusion the main fusion reaction in the sun-, and iii) neutrino - deuteron reactions, which are the detection reactions used by the Sudbury Neutrino Observatory 25. Hence, via the absolute neutrino rates, the precise $\mu \mathrm{d}$ capture rate determination would 'calibrate' the sun. Existing experimental results on $\Lambda_{d}$ are not precise enough (Fig 4 a) and also using other sources leaves the present experimental knowledge on $L_{1 A}$ rather sparse, as shown in Fig $4 \mathrm{p}$. With precision neutrino physics on the horizon the precise knowledge of $L_{1 \mathrm{~A}}$ will be necessary, as it also influences the determination of $\delta m_{21}^{2}$ and $\Theta_{13}[26$.

The experimental principle of MuSun will follow the successful MuCap approach, but for control and optimization of muonic molecule formation and muon catalyzed fusion reactions in deuterium one has to use a high density cryo-target at $\sim 30 \mathrm{~K}[22[23$. $d d$ fusion reactions occur at much higher rates than muon capture and hence represent a severe background, but also allow to monitor the hyperfine populations of the muonic atom 24. High $Z$ target purity will be even more critical as in MuCap. In a first engineering run a new pad-based TPC was successfully tested with high purity deuterium in late 2008.

\section{Further fundamental measurements and ideas}

There are several other precision measurements of fundamental parameters ongoing or under discussion at PSI, which either will test the Standard Model or search for new physics.

- The muonic Lamb-shift experiment [27] is preparing for its first physics data taking in 2009 , and wants to precisely determine the proton charge radius via observation of the $2 \mathrm{p}-2 \mathrm{~s}$ energy difference in muonic hydrogen.

- A precise test of the electron-muon universality is being performed within the PEN experiment and corresponding data are being analyzed [28.

- The search for the lepton-flavor-violating process $\mu \rightarrow$ eee would be a sensitive search for new physics and complement the present MEG activity. There is an ongoing dis- 
cussion on 2 suggested experimental approaches, how to obtain a sensitivity which improves the present experimental limit by roughly 3 orders of magnitude [29].

- A sensitive search for a CP violating muon electric dipole moment (EDM) was suggested in 30 using a compact storage ring, which could make use of PSI's high muon intensity and reach a sensitivity of $5 \times 10^{-23} \mathrm{e} \cdot \mathrm{cm}$. In this way it would test' new physics' and pave the way for higher sensitivity tests of muon and other charged particle EDMs.

- The discussion about dark matter and dark energy has also put interest in particles decaying into mirror worlds, other dimensions or to other branes. Hence the decay products would be invisible. A search for the invisible decay of muons was suggested in [31, and might be also searched for by using the MuCap setup.

- High brightness muon beams would also allow a first test of the gravitational interaction of antimatter of a purely leptonic system, which involves second generation particles, namely muonium $\left(\mu^{+} e^{-}\right)[32$.

Given these and more ideas, one can be sure that precision measurements using muons, at PSI and other facilities in the world, will also in future contribute to a deeper understanding and testing of the Standard Model and provide a fair chance to first find 'new physics' beyond our presently accepted theory.

\section{Acknowledgements}

I would like to express my sincere gratitude for advice, support and many stimulating discussions to K. Kirch, P. Kammel, D. Hertzog, S. Ritt, C. Casella, C. Petitjean, M. Seidel, and especially all members of the MuCap, MuLan and MuSun Collaborations.

\section{References}

[1] W. Wagner et al., Nucl. Instr. and Meth. A (2008) in press.

[2] L.M. Barkov et al., Search for $\mu^{+} \rightarrow e^{+} \gamma$ down to $10^{-14}$ branching ratio, The MEG experiment, PSI proposal R-99-05, 1999; G. Signorelli, these proceedings.

[3] M.L. Brooks et al., Phys. Rev. Lett. 83 (1999) 1521.

[4] W.J. Marciano, Phys.Rev.D 60 (1999) 093006.

[5] T. van Ritbergen, R.G. Stuart, Nucl. Phys. B 564 (2000) 343; T. van Ritbergen, R.G. Stuart, Phys. Lett. B 437 (1998) 201.

[6] A. Pak, A. Czarnecki, Phys. Rev. Lett. 100 (2008) 241807.

[7] D.B. Chitwood, et al. (MuLan Collaboration), Phys. Rev. Lett. 99 (2007) 032001.

[8] A. Barczyk et al., Phys. Lett.B 663 (2008) 172.

[9] J. Duclos et al., Phys. Lett. B47 (1973) 491; M.P. Balandin et al., Sov. Phys. JETP 40 (1974) 811; G. Bardin et al., Phys. Lett. B137 (1984) 135; K. Giovanetti et al., Phys. Rev. D29,(1984) 343.

[10] P. Ackerbauer et al., Phys. Lett. B417 (1998) 224.

[11] D.H. Wright et al., Phys. Rev. C 57 (1998) 373.

[12] A. Andreev et al. (MuCap Collaboration), Phys. Rev. Lett. 99 (2007) 032002.

[13] V. Bernard, L. Elouadrhiri, and U.-G. Meissner, J. Phys. G 28 (2002) R1.

[14] G. Bardin et al., Nucl. Phys. A352 (1981) 365.

[15] G. Bardin et al., Phys. Lett. B 104, 320 (1981) 320; J.H.D. Clark et al., Phys. Rev. Lett. 96 (2006) 073401.

[16] D.D. Bakalov et al., Nucl. Phys. A384, 302 (1982) 302. 
[17] P. Kammel in: Proceedings of the EXA'02 - International Workshop on Exotic Atoms - Future Perspectives, Vienna, Austria, 2002; Austrian Academy of Sciences Press, Vienna, 2003; arXiv:nuclex/0304019 B. Lauss in: Proceedings of the EXA'05 - Intern. Conference on Exotic Atoms, Vienna, Austria, 2005, Austrian Academy of Sciences Press, Vienna 2005; arXiv:nucl-ex/0401005

[18] V.A. Ganzha et al., Nucl. Instr. and Meth. A578 (2007) 485,

[19] V. Bernard, T.R. Hemmert, U.-G. Meissner, Nucl. Phys. A686, (2001) 290; S. Ando, F. Myhrer, K. Kubodera, Phys. Rev. C63 (2000) 015203.

[20] A. Czarnecki, W.J. Marciano, A. Sirlin Phys. Rev. Lett. 99 (2007) 032003.

[21] S. Ando et al., Phys. Lett. B 533 (2002) 25; J.W. Chen et al., Phys. Rev. C 72 (2005) 061001.

[22] V.A. Andreev et al., Muon Capture in deuterium, the MuSun experiment, PSI Proposal R-08-01, 2008.

[23] C. Petitjean in: Proceedings of the International Symposium on Pulsed Neutron and Muon Sciences, Mito, Japan 2008; Nucl. Inst. and Meth. A 2008, in press.

[24] C. Petitjean et al., Hyperfine Int. 118 (1999) 127; B. Lauss et al., Hyperfine Int. 118 (1999) 79.

[25] B. Aharmim et al., Phys. Rev. C75 (2007) 045502; J.-W. Chen, K.M. Heeger, R.G. Hamish Robertson, Phys.Rev. C67 (2003) 025801.

[26] A.B. Balantekin, H. Yuksel, Phys. Rev. C68 (2003) 055801 and Int.J. Mod. Phys., E14 (2005) 39.

[27] R. Pohl et al., AIP Conf. Proc. 796 (2005) 253.

[28] D. Poĉanić, these proceedings.

[29] A. van der Schaaf, CHIPP Meeting, Lausanne, Switzerland, 2008.

[30] A. Adelmann et al., arxiv:hep-ex/0606034

[31] S. Gninenko, Phys. Rev. D76 (2007) 055004.

[32] K. Kirch, arxiv:hep-ex/0702143 